\title{
The controversial stability analysis
}

\author{
Ercília Sousa * \\ Departamento de Matemática, Universidade de Coimbra, \\ Apartado 3008, 3000 Coimbra, Portugal. E-mail: ecs@mat.uc.pt.
}

November 12, 2002

\begin{abstract}
In this review we present different techniques for obtaining stability limits for a finite difference scheme - the forward-time and space-centred numerical scheme applied to the convection-diffusion equation. A survey of past attempts to state stability conditions for this scheme illustrates the difficulties in stability analysis that arise as soon as a scheme becomes more complex and illuminates the concepts of necessary and sufficient conditions for stability.
\end{abstract}

\section{Introduction}

Stability is an essential concern when one is studying the convergence of a numerical scheme and it is often very difficult to obtain stability limits for a numerical scheme. When we are getting stability conditions for a numerical scheme, it is crucial to know whether they are necessary, sufficient, or necessary and sufficient stability conditions, since misunderstandings concerning this point can easily lead to mistaken conclusions, as we shall see later.

The study of the stability of the forward-time centred-space numerical scheme applied to the convection-diffusion equation (also called the central scheme) starts in 1964 with the work of Fromm (1). Fromm applied the von Neumann analysis and derived stability limits for the two dimensional vorticity transport equation. For the sake of clarity, in this paper we shall instead present the corresponding results for a one-dimensional problem. While trying to obtain the stability limits Fromm committed a mistake that led him to derive sufficient stability conditions that were not necessary ones. Nevertheless, these were assumed by Fromm and others to be both necessary and sufficient conditions for stability and therefore needlessly restrictive conditions were taken in the literature to be the correct necessary and sufficient conditions.

Four years later, in 1968, Hirt (2) derived stability conditions based on the examination of truncation errors and the CFL condition. These techniques only give us necessary conditions for stability, although it was later proven that the

\footnotetext{
${ }^{*}$ This work was partially supported by CMUC/FCT
} 
conditions obtained by Hirt were in fact stability limits that are both necessary and sufficient.

In 1972, Roache (3) presented the analyses of Fromm and Hirt in a textbook chapter, assuming wrongly that Fromm's analysis provides necessary and sufficient conditions for stability whereas Hirt's provides conditions that are only necessary. Following this publication, a considerable number of articles repeated the error of taking Fromm's stability limits to be necessary and sufficient conditions (for instance, (4),(5),(6)).

In 1978 Siemienieniuch and Gladwell (7) applied the criterion of the spectral radius for stability and arrived at completely different conditions from those of Fromm and Hirt. This difference is not surprising since the spectral radius of the matrix iteration only gives us necessary conditions for stability.

One year later, in 1979, Rigal (8) used von Neumann analysis and gave a geometrical proof to derive the stability limits. Rigal obtained the correct limits, and these were proven to be necessary for stability. In 1981, Clancy (9) obtained the same stability limits by using the von Neumann analysis and an analytical approach, but could only prove that they are sufficient for stability. It is only in 1984 that Hindmarsh et al (10) used the von Neumann analysis to finally prove that the correct limits are necessary and sufficient stability conditions.

Although it might have been expected that these results would have clarified the confusion, we nevertheless find recent papers, such as Weinan and Liu (11) in 1994, presenting sufficient conditions and wrongly assuming that they imply the correct necessary and sufficient stability conditions.

The content of the paper is organised as follows. In section 2 we introduce the model problem, the central numerical scheme and a short summary about the relation between convergence and stability. In section 3 we give an overview of the different tools for stability analysis that were considered by the different authors and in section 4 we give the detailed historical review that includes the main proofs to achieve stability conditions.

\section{Model problem and finite difference scheme}

Consider the linear convection-diffusion equation

$$
\frac{\partial u}{\partial t}(x, t)+V \frac{\partial u}{\partial x}(x, t)=D \frac{\partial^{2} u}{\partial x^{2}}(x, t), \quad x>0, t>0,
$$

which describes the convection and the diffusion of a function $u(x, t)$. The convection velocity $V>0$ and the diffusion $D>0$ are assumed constant. Suppose the equation (1) is subject to the initial condition

$$
u(x, 0)=u_{0}(x), \quad x>0,
$$

and to the boundary condition,

$$
u(0, t)=0, \quad t>0 .
$$


Solutions of this equation are bounded and otherwise well-behaved. We assume this problem is properly posed from the outset, in such a way that existence and uniqueness of solutions are ensured under physically reasonable assumptions.

Suppose we have approximations $U_{j}^{n}$ to the values $u\left(x_{j}, t_{n}\right)$ at the mesh points

$$
x_{j}=j \Delta x, \quad j \in \mathbb{N} \quad \text { and } \quad t_{n}=n \Delta t, \quad n \in \mathbb{N}
$$

where $\Delta x$ is the space step and $\Delta t$ denotes the time step.

We use the usual central and second difference operators,

$$
\Delta_{0} U_{j}:=\frac{1}{2}\left(U_{j+1}-U_{j-1}\right), \quad \text { and } \quad \delta^{2} U_{j}:=U_{j+1}-2 U_{j}+U_{j-1} .
$$

Assume that the initial value problem (1),(2) is approximated by the central numerical scheme

$$
U_{j}^{n+1}=U_{j}^{n}-\nu \Delta_{0} U_{j}^{n}+\mu \delta^{2} U_{j}^{n},
$$

where

$$
\nu=\frac{V \Delta t}{\Delta x}, \quad \mu=\frac{D \Delta t}{\Delta x^{2}} .
$$

The questions about stability and accuracy of a finite difference method are directly related to the convergence property of the numerical method as stated in the important Lax equivalence theorem.

Before enunciate the Lax equivalence theorem firstly we give the definitions of stability and consistency for the finite difference scheme (4).

Definition 2.1 The finite difference method (4) is called stable in the norm $\|\cdot\|$ if there exist constants $K$ and $c$ such that

$$
\left\|U^{n}\right\| \leq K e^{c n \Delta t}|| U^{0}\left\|=K e^{c t_{n}}\right\| U^{0} \|
$$

where $t_{n}=n \Delta t$, and $K>0$ and $c$ are independent of the space step and time step.

Definition 2.2 The finite difference scheme (4) is consistent up to time $T_{0}$ in the norm $\|\cdot\|$ with equation (1) if the actual solution $u$ to the initial value problem (1)-(3) satisfies

$$
u_{j}^{n+1}=\left[1-\nu \Delta_{0}+\mu \delta^{2}\right] u_{j}^{n}+\Delta t T^{n},
$$

where $u_{j}^{n}=u(j \Delta x, n \Delta t),\left\|T^{n}\right\| \leq \tau(\Delta x), n \Delta t<T_{0}$ and $\tau(\Delta x) \rightarrow 0$ as $\Delta t \rightarrow 0$. Here is assumed that $\Delta x$ is defined in terms of $\Delta t$ and goes to zero with $\Delta t$.

Theorem 2.3 Lax Equivalence Theorem (see Richtmyer and Morton (13)): Given a properly posed initial-value problem for a linear partial differential equation and a linear finite difference approximation to it that satisfies the consistency condition, stability is the necessary and sufficient condition for convergence. 


\section{Stability analysis - necessary and sufficient condi- tions}

In this section we give an overview of three tools that can be used to prove stability of a numerical finite difference scheme: von Neumann analysis, CFL condition and the matrix method.

\section{1 von Neumann stability analysis}

The von Neumann (Fourier) method is the most well known classical method to determine necessary and sufficient stability conditions. If we assume periodic boundary conditions the von Neumann analysis is based on the decomposition of the numerical solution into a Fourier series as

$$
U_{j}^{n}=\sum_{p=-N}^{N} \kappa_{p}^{n} \mathrm{e}^{\mathrm{i} \xi_{p}(j \Delta x)}
$$

where $\quad \mathrm{i}=\sqrt{-1}, \quad \kappa_{p}^{n}$ is the amplification factor of the $p$-th harmonic and $\xi_{p}=\frac{p \pi}{N \Delta x}$. The product $\xi_{p} \Delta x$ is often called the phase angle:

$$
\theta=\xi_{p} \Delta x
$$

and covers the domain $[-\pi, \pi]$ in steps of $\pi / N$. The region around $\theta=0$ corresponds to the low frequencies while the region $\theta=\pi$ is associated with the high-frequencies. In particular, the value $\theta=\pi$ corresponds to the highest frequency resolvable on the mesh, namely the frequency of wavelength $2 \Delta x$.

Considering a single mode, $\kappa^{n} \mathrm{e}^{\mathrm{i} j \theta}$, its time evolution is determined by the same numerical scheme as the complete numerical solution $U_{j}^{n}$. Hence inserting a representation of this form into a numerical scheme we obtain a stability condition by imposing an upper bound to the amplification factor, $\kappa$.

Definition 3.1 The amplification factor is said to satisfy the von Neumann condition if there is a constant $K$ such that

$$
|\kappa(\xi)| \leq 1+K \Delta t, \quad \forall \xi \in \mathbb{R} .
$$

Consider a function $U$ defined in a discrete set of points $x_{j}=j \Delta x, U_{j}=$ $U(j \Delta x)$. The Euclidean or $l_{2}$-norm is defined to be $\|U\|_{2}=\sqrt{\sum_{j} \Delta x U_{j}^{2}}$. We have the following theorem, the proof of which can be found for instance in Sod (12):

Theorem 3.2 A two level linear finite difference method is stable in the $l_{2}$-norm if and only if the von Neumann condition is satisfied.

However, for some problems the presence of the arbitrary constant in (5) is too generous for practical purposes, although being adequate for eventual convergence in the limit $\Delta t \rightarrow 0$. In practice, the inequality (5) is substituted by the following stronger condition,

$$
|\kappa(\xi)| \leq 1, \quad \forall \xi \in \mathbb{R}
$$


or in terms of the phase angle,

$$
|\kappa(\theta)| \leq 1, \quad \forall \theta \in[-\pi, \pi] .
$$

This has been called practical stability by Richtmyer and Morton (13) or strict stability by other authors. In some cases condition (5) allows numerical modes to grow exponentially in time for finite values of $\Delta t$. Therefore, the practical, or strict, stability condition (6) is recommended in order to prevent numerical modes from growing faster than the physical modes of the differential equation.

\subsection{The CFL stability condition}

The CFL condition is named after Courant, Friedrichs and Lewy. They used finite difference methods as an analytic tool for proving the existence of solutions of certain partial differential equations - see (14) and later the English translation (15). It was in this paper that the concept of stability was first introduced. The idea is to define a sequence of approximate solutions using finite difference equations, prove that they converge as the grid is refined, and then show that the limit function must satisfy the partial differential equation giving the existence of a solution. When proving the convergence of this sequence they recognized a necessary condition for stability of the numerical method since what they were looking for is what we look for when trying to find a stable method.

CFL stability condition: A numerical method can be convergent only if its numerical domain of dependence contains the true domain of dependence of the partial differential equation, at least in the limit as $\Delta t$ and $\Delta x$ go to zero.

Recall the definition of domain of dependence of a partial differential equation: Suppose we have a fixed point $(\mathbf{x}, \mathbf{t})$ in space and time and that the solution at this point, $u(\mathbf{x}, \mathbf{t})$, depends only of the initial solution $u_{0}$ at $m$ particular points. The set of these $m$ points is called the domain of dependence. The numerical domain of dependence of a method can be defined in a similar manner as the set of points where the initial data can possibly affect the numerical solution at a point $\left(\mathbf{x}_{\mathbf{j}}, \mathbf{t}_{\mathbf{n}}\right)$.

Note that the CFL condition is only a necessary condition for stability and not necessarily sufficient.

\subsection{The matrix method}

The explicit method we are considering can be written in the form of a matrix iteration, where the nodal points are $U_{j}^{n}, j=1, \ldots, N$. We assume that we have Dirichlet boundary conditions, this is,

$$
U_{0}^{n}=U_{N+1}^{n}=0, \quad \forall n .
$$

The choice of this outflow boundary is motivated by the fact that we assume that the exact solution goes to zero when $x$ goes to infinity.

Introducing the vector $U^{n}=\left\{U_{1}^{n}, \ldots, U_{N}^{n}\right\}^{T}$, the scheme may be written as a matrix equation

$$
U^{n+1}=A U^{n}, \quad n=0,1,2, \ldots
$$


where $A$ is an $N \times N$ matrix.

Any errors $E^{n}$ in a calculation based on (9) will grow according to

$$
E^{n+1}=A E^{n}, \quad n=0,1,2, \ldots
$$

where $E^{n}=u^{n}-U^{n}$ with $u^{n}, U^{n}$ the exact and numerical solutions of (9), respectively, at $t=n \Delta t$.

Given $A \in \mathbb{R}^{N \times N}$ denote the spectral radius of $A$ by $\rho(A)$ and the $L_{2}$-norm of the matrix $A$ by $\|A\|$. We recall that

$$
\|A\|=\rho(A) \quad \text { if } \quad A \in \mathbb{R}^{N \times N} \quad \text { is normal. }
$$

It is well known that for any $A \in \mathbb{R}^{N \times N}$

$$
A^{m} \rightarrow 0 \quad \text { as } \quad m \rightarrow \infty \quad \text { if and only if } \rho(A)<1,
$$

and that

$$
\rho(A) \leq\|A\| .
$$

A simple criterion for regulating the error growth governed by (10) is given by

$$
\rho(A) \leq 1 .
$$

When the matrix $A$ is not normal the spectral radius gives no indication of the magnitude of $E^{n}$ for finite $n$. In this case a condition of the form $\rho(A)<1$ guarantees eventual decay of the solution, but does not control the intermediate growth of the solution. Then, it is easy to understand that the condition (11) is a necessary condition for stability but not always sufficient.

\section{The historical events}

Stability analysis is often tricky and difficult. The history of attempts to state stability conditions for the central scheme (4) illustrates some of the difficulties that appears in stability analysis.

A first von Neumann stability condition for the central scheme was derived in 1964 in a paper by Fromm (1) when determining the stability limits for the twodimensional vorticity transport equation. What follows is the one-dimensional equivalent of Fromm's two-dimensional result. Their derivation contains an important error, but in the two-dimensional setting there is also a second distinct error in Fromm's analysis. Before elucidating how the mistake was made, we present Fromm's proof.

Fromm stability analysis (1964): A stability condition for the central scheme is given by

$$
\nu \leq 2 \mu \leq 1 \text {. }
$$

Fromm used von Neumann analysis to determine the stability limits. Consider the amplification factor associated with the central scheme (4),

$$
\kappa(\theta)=1-\mathrm{i} \nu \sin \theta+2 \mu(\cos \theta-1), \quad-\pi \leq \theta \leq \pi .
$$


Let us define $f$, such as, $f(\theta)=|\kappa(\theta)|^{2}$. Then

$$
f(\theta)=[1+2 \mu(\cos \theta-1)]^{2}+\nu^{2} \sin ^{2} \theta .
$$

We seek a maximum of $f$ in terms of $\cos \theta$. Thus

$$
\frac{d f}{d(\cos \theta)}=4 \mu+8 \mu^{2}(\cos \theta-1)-2 \nu^{2} \cos \theta \quad \text { and } \quad \frac{d^{2} f}{d(\cos \theta)^{2}}=8 \mu^{2}-2 \nu^{2} .
$$

Note that a maximum occurs only if $8 \mu^{2}-2 \nu^{2}<0$, that is, if $\nu>2 \mu$. The maximum or minimum occurs for

$$
\cos \theta=\frac{2 \mu(1-2 \mu)}{\nu^{2}-4 \mu^{2}}
$$

or, substituting (15) in (14), for

$$
f(\theta)=\frac{\nu^{2}\left(\nu^{2}+1-4 \mu\right)}{\nu^{2}-4 \mu^{2}} .
$$

The condition of (7) together with (16) thus implies $\nu^{2}-4 \mu^{2} \leq 0$. For $\nu>2 \mu$, this condition cannot be met; hence we conclude that we must first have

$$
\nu \leq 2 \mu
$$

While no maximum occur if (17) is satisfied, we must still consider the end values of $\cos \theta$. Thus if $\cos \theta=1$, then $f(\theta)=1$ and stability is indicated. While $\cos \theta=-1$ we arrive at the condition

$$
2 \mu \leq 1
$$

We therefore have the stability condition specified in (12).

Note that Fromm does a very basic error, when substitutes $\cos \theta$ in $f$, respectively defined by (15) and (14), obtaining (16) and seek the maximum of this function with the only restriction, $\nu>2 \mu$. When we seek the maximum of $f$ in terms of $\cos \theta$ and we get (16), this is only a valid step for $\mu, \nu$ such that $|\cos \theta| \leq 1$. Since at this stage of the proof we were assuming $\nu>2 \mu$ we can easily check that $|\cos \theta| \leq 1$ if and only if $\nu^{2} \geq 2 \mu$. In that case, for the set $\left\{(\mu, \nu): \nu>2 \mu, \nu^{2} \leq 2 \mu\right\}$, see figure 1, nothing can be inferred from (16).

The condition (12), displayed in figure 2, was many times reported in the literature as a necessary and sufficient condition, although it is only a sufficient condition for stability. When condition (17) is imposed, this is a sufficient condition to have $f(\theta) \leq 1$.

Four years later in 1968, Hirt derived necessary stability conditions for the central scheme but did not mention Fromm's paper. The method used, was based on the examination of a certain kind of truncation errors and also on the application of the CFL stability condition mentioned in section 2.2 .

Hirt's stability analysis (1968): The stability conditions are

$$
2 \mu \leq 1, \quad 2 \mu \geq \nu^{2} .
$$




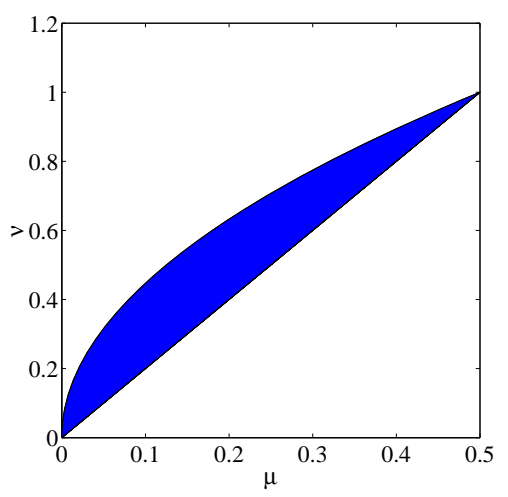

Figure 1: The set of values of $\mu$ and $\nu$ that Fromm did not consider in the stability analysis.

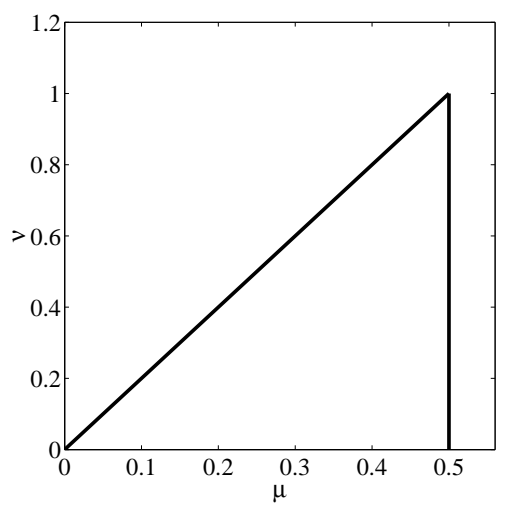

Figure 2: Fromm's stability analysis (1964).

Consider each term in (4) as a continuous function of $x$ and $t$. For example, consider $U_{j+1}^{n}$ as denoting $U(x+\Delta x, t)$, that is, the solution approximating $u(x+$ $\Delta x, t)$. Replace $U$ by $u$ and expand each term of (4) in a Taylor series about the point $(x, t)$ to obtain

$$
\frac{\partial u}{\partial t}+V \frac{\partial u}{\partial x}-D \frac{\partial^{2} u}{\partial x^{2}}=-\frac{1}{2} \Delta t \frac{\partial^{2} u}{\partial t^{2}}+O\left(\Delta x^{2}, \Delta t^{2}\right) .
$$

Thus, (20) is approximated by keeping only the lowest-order even and odd derivative terms, which are

$$
\frac{1}{2} \Delta t \frac{\partial^{2} u}{\partial t^{2}}+\frac{\partial u}{\partial t}+V \frac{\partial u}{\partial x}-D \frac{\partial^{2} u}{\partial x^{2}}=0
$$

It is significant that (21) is not identical to (1). In fact, it is the difference between these equations that accounts for the computational instabilities of (4). To see this, recall that the difference equation propagates information into a region bounded by lines whose slopes are

$$
\frac{d x}{d t}= \pm \frac{\Delta x}{\Delta t}
$$


The $\Delta t$ term in (21) makes the equation hyperbolic with characteristic lines whose slopes are

$$
\frac{d x}{d t}= \pm\left(\frac{2 D}{\Delta t}\right)^{1 / 2}
$$

If the difference equation is to have solutions approximating those of (21), then its region of influence must include the region of influence of (21) - see CFL stability condition.

The necessary condition is

$$
\frac{2 D}{\Delta t} \leq\left(\frac{\Delta x}{\Delta t}\right)^{2}
$$

The condition (22) is identical to the first stability condition in (19).

The second stability condition is also obtained from (21), if the term proportional to $\Delta t$ is rewritten. From (20) we find that

$$
\frac{\partial^{2} u}{\partial t^{2}}=V^{2} \frac{\partial^{2} u}{\partial x^{2}}-2 V D \frac{\partial^{3} u}{\partial x^{3}}+D^{2} \frac{\partial^{4} u}{\partial x^{4}}=0
$$

Combining (23) and (21), and neglecting terms of second order in $\Delta t$

$$
\frac{\partial u}{\partial t}+V \frac{\partial u}{\partial x}=\left(D-\frac{1}{2} V^{2} \Delta t\right) \frac{\partial^{2} u}{\partial x^{2}}+V D \Delta t \frac{\partial^{3} u}{\partial x^{3}}-\frac{1}{2} D^{2} \Delta t \frac{\partial^{4} u}{\partial x^{4}}
$$

The last two terms in (24) can be dropped, as before, since they are high-order derivatives, so that

$$
\frac{\partial u}{\partial t}+V \frac{\partial u}{\partial x}=\left(D-\frac{1}{2} V^{2} \Delta t\right) \frac{\partial^{2} u}{\partial x^{2}}
$$

Comparing this result with (1) we now find there is an additional diffusion term. When (25) has a negative coefficient it has solutions that grow exponentially in time. For non-growing solutions, stable solutions, it is necessary that

$$
D \geq \frac{1}{2} V^{2} \Delta t
$$

This is the second condition in (19).

Hirt also wrote that a study of the (13) shows that the magnitude of $\kappa$ is less than unity for all $\xi$ if the two conditions in (19) are satisfied. However, no proof was given involving the amplification factor. The necessary conditions derived by Hirt are displayed in figure 3 and as we shall see later these are the correct necessary and sufficient stability limits. For a graphical illustration of the mistake of Fromm compare figures 1-3.

Four years later in $\mathbf{1 9 7 2}$ Roache (3) compiles in a book chapter, Fromm's analysis and Hirt's analysis. He writes that if the condition $\nu \leq 2 \mu$ is combined with the diffusion restriction $2 \mu \leq 1$, the result is $\nu^{2} \leq 2 \mu$. In fact the first ones imply the third one but they are not equivalent. Additionally he mentioned that the conditions obtained by Fromm are necessary and sufficient stability conditions and those obtained by Hirt's are only necessary conditions. He wrote: "This 
analysis (talking about Hirt's analysis) does not uncover the condition $\nu \leq 2 \mu$, and is therefore seen to provide necessary but not sufficient conditions for stability of the model advection-diffusion equation". As we have seen Hirt only assures that the conditions obtained are necessary for stability and Fromm only arrived to sufficient conditions. Neither were proved to be necessary and sufficient conditions.

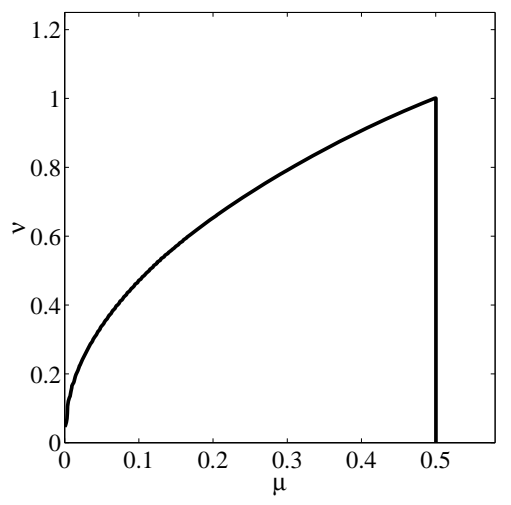

Figure 3: Hirt's necessary stability conditions (1968).

The numerical stability of Fromm's method is many times mentioned in the literature as to involve a restriction on mesh size which can not always be met, for practical reasons - see for instance Torrance et al (1972) (4). Also Marshall (5) in $\mathbf{1 9 7 4}$ gives a linear stability analysis of the central scheme that is the same as Fromm's, although his article does not explain how the linear stability conditions are derived. In 1976, Chien (6) writes a paper which recommends an approach where, unlike with Fromm's analysis, there is no apparent stability limitations on grid size and time step. Consequently, the main advantage of Chien's approach loses its interest once it is compared with the right stability limits.

In an investigation of the stability of the explicit central differenced convectiondiffusion equation, in $\mathbf{1 9 7 8}$ Siemieniuch and Gladwell (7) applied a matrix method with a criterion on the spectral radius for stability. This analysis only lead us to necessary conditions as clarified in section 2.3 .

Siemieniuch and Gladwell stability analysis (1978): The stability conditions are:

$$
\begin{cases}0<\mu<\frac{1}{1+\sqrt{1-\alpha^{2}}}, & \alpha \leq 1 \\ 0<\mu \leq \frac{1}{\alpha^{2}} & \alpha \geq 1,\end{cases}
$$

where $\quad \alpha=\frac{\nu}{2 \mu}$.

The criterion of the spectral radius $\rho(A) \leq 1$ is used to determine the stability limits.

The $N \times N$ matrix $A=\left(a_{i, j}\right)$ associated with the central scheme, for Dirichlet conditions, is a tridiagonal matrix with entries given by

$$
\begin{array}{ll}
a_{i, i}=1-2 \mu, \quad i=1, \ldots, N & a_{i, i-1}=\mu+\nu / 2, \quad i=2, \ldots, N \\
a_{i, i+1}=\mu-\nu / 2, \quad i=1, \ldots, N-1, &
\end{array}
$$


We have, for $\alpha=\nu / 2 \mu$, that the eigenvalues $\lambda_{j}(A)$ are

$$
\lambda_{j}(A)=1-2 \mu+2 \mu \sqrt{\left(1-\alpha^{2}\right)} \cos (j \pi /(N+1)), \quad j=1, \ldots, N
$$

and all the eigenvalues are lower than one in modulus, if $-1 \leq \lambda_{j}(A) \leq 1$. Since the maximum and minimum values of $\lambda_{j}(A)$ are obtained when cosine is -1 or 1 we have two conditions, when $\lambda_{j}$ is real,

$$
-1 \leq 1-2 \mu\left(1+\sqrt{1-\alpha^{2}}\right) \quad \text { and } \quad 1-2 \mu\left(1-\sqrt{1-\alpha^{2}}\right) \leq 1 .
$$

That is,

$$
0<\mu \leq \frac{1}{1+\sqrt{1-\alpha^{2}}} \quad \text { for } \quad \alpha \leq 1 .
$$

If $\alpha>1$, the eigenvalues (28) are complex and the condition $\left|\lambda_{j}(A)\right|<1$ is satisfied for

$$
(1-2 \mu)^{2}+4 \mu^{2}\left(\alpha^{2}-1\right)<1
$$

or

$$
0<\mu \leq \frac{1}{\alpha^{2}}, \quad \text { for } \quad \alpha>1 .
$$

For $\alpha=1$, we have a multiple eigenvalue $\lambda_{j}(A)=1-2 \mu$, leading to the condition $\mu<1$. Then, we have the condition (27). We plot these conditions in figure 4 .

The conditions derived by Siemieniuch and Gladwell (7) are clearly distinct from the stability conditions obtained by Hirt (2) or Fromm (1). Siemieniuch and Gladwell (7) did some numerical experiments but did not explain the discrepancies they observed between their derived stability limits and the inaccuracy or instability of their computed results. The conditions they derived are only necessary conditions for stability and therefore they describe a region where instabilities may still occur.

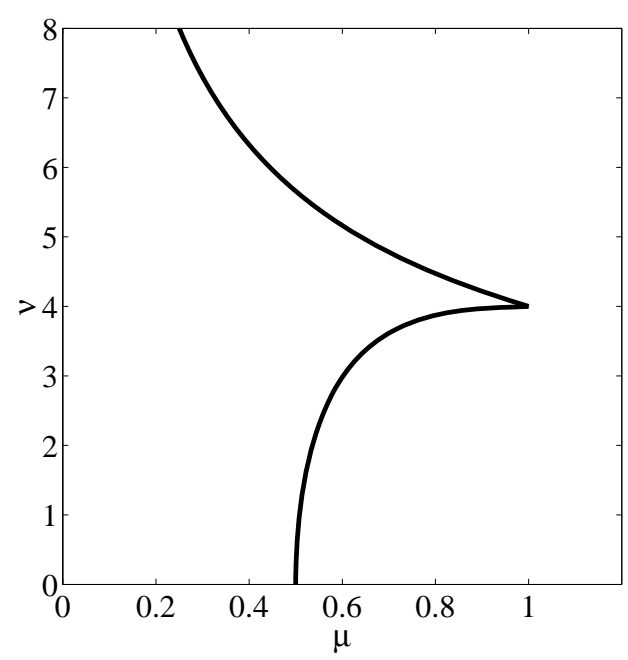

Figure 4: Siemieniuch and Gladwell stability analysis (1978). 
Rigal in 1979 gives a proof based in the geometrical interpretation of the amplification factor and as Hirt, Rigal presents the right stability limits only proving they are necessary conditions for stability.

Rigal stability analysis (1979): Consider the amplification factor, $\kappa(\theta)$, (13). To guarantee the stability of the scheme we write $\sup _{\theta \in[-\pi, \pi]}|\kappa(\theta)|^{2} \leq 1$ for,

$$
|\kappa(\theta)|^{2}=[1-2 \mu+2 \mu \cos \theta]^{2}+\nu^{2} \sin ^{2} \theta .
$$

We can obtain the result geometrically: from (29) we deduce that $|\kappa(\theta)|^{2}$ describes, in the complex plane, the upper half of an ellipse $\mathrm{E}$ with centre $(1-2 \mu, 0)$ and semiaxes $2 \mu$ and $\nu$, when $\theta$ belongs to $[0, \pi]$. Then the stability condition prescribes that $\mathrm{E}$ is contained within the unit-circle $\mathrm{C}$ - see figure 5 .

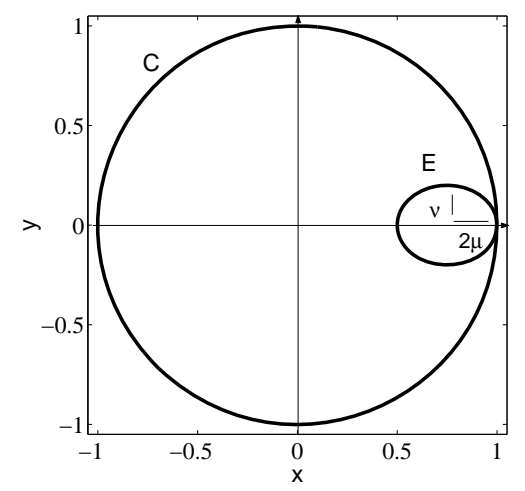

Figure 5: Polar diagram illustrating the amplification factor. If the ellipse lies entirely on and within the unit circle, the numerical scheme is stable.

For the $x$-axis we have a first condition

$$
2 \mu \leq 1
$$

Then we write the cartesian equations of the circle $\mathrm{C}$ and the ellipse $\mathrm{E}$ as

$$
x^{2}+y^{2}=1, \quad \frac{(x-1+2 \mu)^{2}}{4 \mu^{2}}+\frac{y^{2}}{\nu^{2}}=1 .
$$

We prescribe that they have only one intersection, the point $B(1,0)$, to imply that there is no intersection between the two curves next to this point. Eliminating $y$ between the two equations (31) we obtain

$$
x=1 \quad \text { or } \quad x=\frac{(1-\mu) \nu^{2}+4 \mu^{2}}{\nu^{2}-4 \mu^{2}} \quad \text { and } \quad y^{2}=1-x^{2} .
$$

We must have $x \geq 1$ otherwise an intersection point with $y^{2} \leq 1-x^{2}$ would exist. Hence we should have,

$$
\nu^{2} \leq 2 \mu
$$

Consequently (30) and (32) are the conditions such that the Fourier components and therefore the amplitude errors will be bounded. 
Note that if we observe figure 5 we can easily see that Fromm's condition, $\nu \leq 2 \mu \leq 1$, is a sufficient condition for stability.

In 1980 two papers appear commenting the paper of Siemieniuch and Gladwell, one by Griffiths et al. (16) and the other one by Morton (17). Griffiths et al explains how the stability techniques used in Siemieniuch and Gladwell have certain ambiguities in the control of the error growth. Morton clarifies that most of the stability analysis done by Siemieniuch and Gladwell was based in the matrix method and an associated concept of stability, which can be misleading in both theory and practice for such problems.

Also in 1980 Leonard (18) gives a geometrical interpretation similar to Rigal proving that the condition $2 \mu \leq 1$ is a necessary condition for stability and that $\nu^{2} \leq 2 \mu$ is a sufficient stability condition.

Clancy in $\mathbf{1 9 8 1}$ proves correct stability limits to be sufficient conditions for stability. Although Hirt and Rigal presented also the correct limits, they were only proved to be necessary for stability.

Clancy's stability analysis (1981): The complex amplification factor for the central scheme (4) is given by (13), whose magnitude must be everywhere less than or equal to one for the numerical scheme to be stable.

Setting $|\kappa(\theta)|^{2} \leq 1$ yields

$$
-4 \mu(1-\cos \theta)+4 \mu^{2}(1-\cos \theta)^{2}+\nu^{2}(1-\cos \theta)(1+\cos \theta) \leq 0 .
$$

For $\theta=0$ the inequality (33) is satisfied for all $\nu$ and $\mu$. For $\theta \neq 0$ we divide through by $4 \mu(1-\cos \theta)$ to obtain

$$
\left(\mu+\frac{\nu^{2}}{4 \mu}\right)+\left(-\mu+\frac{\nu^{2}}{4 \mu}\right) \cos \theta \leq 1
$$

To investigate the stability of the scheme using (34) two cases must be identified depending on the sign of the term multiplying $\cos \theta$.

Case I: Suppose $\frac{\nu^{2}}{4 \mu}-\mu \leq 0$. Then we have $\nu^{2} \leq 4 \mu^{2}$. Assuming that the left-hand side of (34) will be a maximum for $\theta=\pi$. Thus, (34) will be satisfied for all $\theta$ if

$$
\mu+\frac{\nu^{2}}{4 \mu}+\mu-\frac{\nu^{2}}{4 \mu} \leq 1
$$

or $\mu \leq 1 / 2$.

Case II: Suppose $\frac{\nu^{2}}{4 \mu}-\mu \geq 0$. This is precisely the case Fromm didn't take in consideration carefully. Under this assumption the left-hand side of (34) approaches a maximum as $\theta$ approaches zero. Thus (34) will be satisfied for all $\theta$ if

$$
\mu+\frac{\nu^{2}}{4 \mu}-\mu+\frac{\nu^{2}}{4 \mu} \leq 1
$$

or $\nu^{2} \leq 2 \mu$. Furthermore, from our assumption in case II we have $4 \mu^{2}<\nu$ which along with $\nu^{2} \leq 2 \mu$ yields $\mu \leq 1 / 2$.

Hindmarsh et al in $\mathbf{1 9 8 4}$ appears to be the first to have proved that the stability conditions are simultaneously necessary and sufficient conditions. They give a 
theorem for a multidimensional convection-diffusion equation, that we present here for the one-dimensional case.

Hindmarsh et al stability analysis (1984): The amplificator factor for the scheme (4) is given by (13). From (13), we have

$$
|\kappa(\theta)|^{2}=[1-2 \mu(1-\cos \theta)]^{2}+[\nu \sin \theta]^{2} .
$$

Necessary condition: We are given $|\kappa(\theta)|^{2} \leq 1$, for all $\theta \in[-\pi, \pi]$. For the case of $\theta=\pi$, we have

$$
|\kappa(\pi)|^{2}=(1-4 \mu)^{2} \leq 1,
$$

and this requires that $2 \mu \leq 1$. For the limiting case $\theta \rightarrow 0$, we can write

$$
|\kappa(\theta)|^{2}=\left[1-2 \mu \frac{\theta^{2}}{2}+O\left(\theta^{4}\right)\right]^{2}+\left[\nu \theta+O\left(\theta^{3}\right)\right]^{2}=1-2 \mu \theta^{2}+\nu^{2} \theta^{2}+O\left(\theta^{4}\right) .
$$

Neglecting high-order terms

$$
|\kappa(\theta)|^{2}=1-\theta^{2}\left(2 \mu-\nu^{2}\right) .
$$

Thus, in order to have $|\kappa(\theta)|^{2} \leq 1,2 \mu-\nu^{2}$ must be non-negative and then we must have $\nu^{2} \leq 2 \mu$.

Sufficient condition: Now assume that we have the conditions

$$
\begin{aligned}
2 \mu & \leq 1 \\
\frac{\nu^{2}}{2 \mu} & \leq 1
\end{aligned}
$$

For $\mu>0$, for arbitrary $\theta$, and using (37), we have

$$
\nu^{2} \sin ^{2} \theta=\frac{\nu^{2}}{2 \mu} 2 \mu \sin ^{2} \theta \leq 2 \mu \sin ^{2} \theta .
$$

Inserting (38) into (35), and denoting $1-\cos \theta$ by $z$, we have

$$
|\kappa(\theta)|^{2} \leq(1-2 \mu z)^{2}+2 \mu\left[1-(1-z)^{2}\right]=1-2 \mu z^{2}+4 \mu^{2} z^{2},
$$

and using condition (36), then $4 \mu^{2} z^{2} \leq 2 \mu z^{2}$. It follows that $|\kappa(\theta)|^{2} \leq 1$.

Thompson (19) in $\mathbf{1 9 8 5}$ by using the same stability criteria as Fromm's, the examination of the critical points of the amplification factor, clarifies that the Fromm's main mistake in one and two dimensions was to introduce an unreal set of solutions by changing the independent variable from $\theta$ to $\cos \theta$.

Although, concerning the stability analysis of the central scheme for the convection-diffusion equation, it might have been expected that from 1984 onwards the confusion has been clarified, we nevertheless find in a more recent paper by Weinan and Liu (11) in $\mathbf{1 9 9 6}$ some inaccurate affirmations.

Weinan and Liu (11) write: "This scheme is stable only under the constraint

$$
\Delta t\left(\frac{V^{2}}{2 D}+\frac{2 D}{\Delta x^{2}}\right)<1
$$




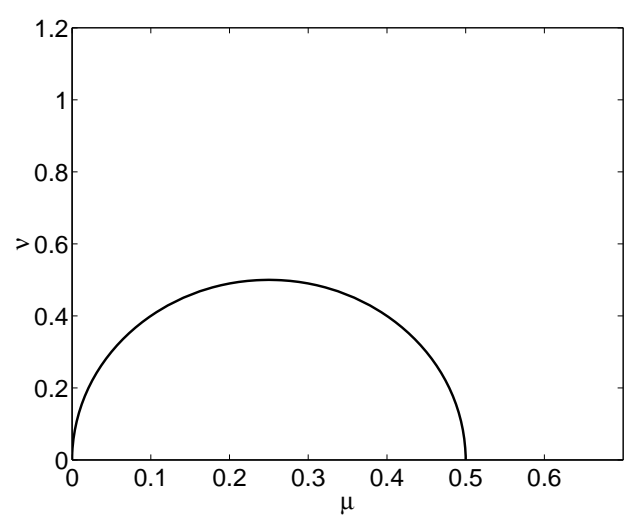

Figure 6: Weinan et al stability analysis (1996)

Therefore, we must have

$$
\frac{D \Delta t}{\Delta x^{2}}<\frac{1}{2} \quad \Delta t<\frac{2 D}{V^{2}} .
$$

Condition (40) can be written as

$$
\frac{\nu^{2}}{2 \mu}+2 \mu<1
$$

and we plot it in figure 6 . This is not the correct stability region. Furthermore from a more restrictive condition (40) they derive a less restrictive condition (41), being the latter a necessary condition to obtain the former but not a sufficient condition.

\section{Conclusion}

The aims of this article were as follows. First, to illustrate the difficulties that can arise in stability analysis. Second, to present different analytic approaches available for obtaining stability conditions. Third, to emphasize the importance of having a full understanding of whether the conditions obtained are sufficient, necessary, or necessary and sufficient for stability.

\section{References}

[1] J. Fromm, The time dependent flow of an incompressible viscous fluid. Methods Comput. Phys. 3:345-382 (1964).

[2] C.W. Hirt, Heuristic stability theory for finite difference equations. J. Comput. Phys. 2:339-355 (1968).

[3] P.J. Roache, Computational Fluid Dynamics. Albuquerque, New Mexico: Hermosa, 1972. 
[4] K. Torrance, R. Davis, K. Eike, P. Gill, D. Gutman, A. Hsui, S. Lyons and H. Zien, Cavity flows driven by buoyancy and shear. J. Fluid Mech. 51:221-246 (1972).

[5] G. Marshall, Computational aspects of a viscous incompressible fluid. Lecture Notes in Phys. 35:269-273 (1974).

[6] J.C. Chien, A general finite difference formulation with application to NavierStokes equations. J. Comput. Phys. 20:268-278 (1976).

[7] J. Siemieniuch and I. Gladwell, Analysis of explicit difference methods for the diffusion-convection equation. Int. J. Numer. Methods Engrg. 12:899-916 (1978).

[8] A. Rigal, Stability analysis of explicit finite difference schemes for the NavierStokes equations. Int. J. Numer. Methods Engrg. 14:617-620 (1979).

[9] R.M. Clancy, A note on finite differencing of the advection-diffusion equation. Mon. Wea. Rev. 109:1807-1809 (1981).

[10] A.C. Hindmarsh, P.M. Gresho and D.F. Griffiths, The stability of explicit Euler time-integration for certain finite difference approximations of the multidimensional advection-diffusion equation. Int. J. Numer. Methods Fluids 4:853897 (1984).

[11] E. Weinan and J-G. Liu, Vorticity boundary condition and related issues for finite difference schemes. J. of Comput. Phys. 124:368-382 (1996).

[12] G.A. Sod, Numerical methods in fluid dynamics: initial and initial boundaryvalue problems, Cambridge University Press: Cambridge, 1988.

[13] R.D. Richtmyer and K.W. Morton, Difference methods for initial-value problems. Wiley-Interscience: New York, 1967.

[14] R. Courant, K.O. Friedrichs and H. Lewy, Uber die partiellen Differenzengleichungen der mathematisches Physik, Math. Ann. 100:32-74 (1928).

[15] R. Courant, K.O. Friedrichs and H. Lewy (1967) On the partial difference equations of mathematical physics, IBM J. 11:215-234 (1967).

[16] D.F. Griffiths, I. Christie and A.R. Mitchell, Analysis of error growth for explicit difference schemes in conduction-convection problems. Int. J. Numer. Methods Engrg 15:1075-1081 (1980).

[17] K.W. Morton, Stability of finite difference approximations to a diffusionconvection equation. Int. J. Numer. Methods Engrg 15, 677-683 (1980).

[18] B.P. Leonard, Note on the von Neumann stability of the explicit FTCS convection diffusion equation. Appl. Math. Modeling 4:401-402 (1980).

[19] H.D. Thompson, B.W. Webb and J.D. Hoffmann, The cell Reynolds number myth. Int. J. Numer. Methods Fluids 5:305-310 (1985). 